\title{
El comercio internacional como multiplicador de la deuda ecológica
}

\author{
Juan Camilo Santamaría Herrera* \\ Evelyn Ivonne Díaz Montaño**
}

Santamaría Herrera, J.C. y Díaz Montaño, E.I. (2011). El comercio internacional como multiplicador de la Deuda ecológica. Revista Activos, 16 y $17,117-134$.

JEL: Q51

Recibido: 7 de abril de 2011 Aprobado: 25 de mayo de 2011

\section{Resumen}

El comercio internacional es ecológicamente desigual, dado que los precios de los productos no equiparan los daños ambientales y sociales de su producción ni los servicios ambientales suministrados inherentemente en el intercambio. El presente documento describe las causas del denominado "efecto escala" del comercio internacional, a partir del concepto de deuda ecológica y de su identificación como multiplicador de la misma. Reconocer la creciente deuda ecológica establece una oportunidad para los países acreedores en la identificación de argumentos a favor, para contribuir en la reivindicación de la soberanía alimentaria y la negociación de

* Contador Público, Especialista en Derecho Económico, estudiante de la Maestría en Medio Ambiente y Desarrollo de la Universidad Nacional de Colombia.

** Contadora Pública, Universidad Nacional de Colombia. Especialista en Gerencia de los Recursos Naturales, Universidad Distrital Francisco José de Caldas. 
la deuda externa. Con ello se busca la solidaridad internacional, la justicia social y una óptima relación entre el comercio internacional y el ambiente.

\title{
Palabras clave
}

Comercio internacional, deuda ecológica, deuda externa, soberanía alimentaria.

Santamaría Herrera, J.C. \& Díaz Montaño, E.I. (2011). The International Commerce like Multiplier of the Ecological Debt. Activos Review, 16 and 17, 117-134.

\begin{abstract}
The international commerce is ecologically unfair, because the products prices dont' equalize the enviromental and social damages that the production involves nor the environmental services given in the process. The present paper describes the causes of the international commerce "scale effect" from the idea of "ecological debt" and its role as multiplier of this debt. To recognize the increasing Ecological Debt stablish an opportunity for the creditor countries to present favorable arguments, contribute in the nutritional sovereignty and the negotiation of foreign debt. All of this aiming to the international solidarity and an ideal relationship between environment and international commerce.
\end{abstract}

\section{Keywords}

International commerce, ecological debt, external debt, food sovereignty.

Santamaría Herrera, J.C. et Díaz Montaño, E.I. (2011). Le commerce international comme multiplicateur de la dette écologique. Revue Activos, 16 et 17, 117-134.

\section{Résumé}

Le commerce international est inégal du point de vue écologique, vu que les prix des produits ne compensent pas les coûts écologiques et sociaux de leur production, ni les services écologiques offerts de façon inhérente dans l'échange. Le présent 
document décrit les causes du l'effet dénommé "effet d'échelle" dans le commerce international, à partir du concept de dette écologique et l'identifie comme son multiplicateur.Reconnaître la dette écologique croissante consiste en une chance pour les pays créanciers, dans l'identification d'arguments en leur faveur, pour la revendication d'une souveraineté alimentaire et la négociation de la dette extérieure, cherchant la solidarité internationale et la justice sociale, en relation avec le commerce international et l'environnement.

\section{Mots clés}

Commerce international, dette écologique, dette extérieure, souveraineté alimentaire.

\section{Introducción}

Dadas las actuales condiciones de degradación ambiental del mundo, analizar hoy en día los conceptos de desarrollo o crecimiento económico es una tarea difícil. Los postulados clásicos que idealizaban el mercado como mecanismo legítimo de crecimiento económico son altamente cuestionables; cuando la discusión trasciende las fronteras nacionales para analizar las relaciones en el comercio internacional, los argumentos en contra son innumerables.

El presente documento tiene como objetivo general identificar los factores que originan un efecto escala multiplicador de la deuda ecológica en la relación comercio internacional-ambiente. A partir de esta consideración se exponen algunos elementos para el establecimiento de soluciones, tales como la aplicación de una estrategia de negociación de la deuda externa o el diseño de una política alimentaria basada en la soberanía de las naciones.

La investigación requirió una revisión y análisis permanente de bibliografía que permitiera soportar de manera conceptual la noción de "deuda ecológica” y presentara los desarrollos teóricos en el tema de comercio internacional y su relación con el ambiente. El documento se subdivide en tres secciones. En primer lugar se plantea la existencia de la deuda ecológica, apartado en el que se abordan el concepto y los elementos de la misma. Posteriormente se desarrolla el efecto escala del comercio 
internacional, se explica su impacto multiplicador en el incremento de la deuda ecológica y se muestra cómo su reconocimiento puede presentar alternativas para su control y mitigación en los países acreedores. Finalmente se establecen conclusiones que exponen de manera resumida los aspectos desarrollados en el trabajo, como puede ser el hecho de que un crecimiento de las transacciones del comercio internacional -que además es desigual-induce al aumento del impacto ambiental a través del incremento en la cantidad de recursos materiales movilizados, al igual que origina un efecto multiplicador de la deuda ecológica.

\section{Marco teórico}

Para desarrollar el tema propuesto en el presente documento es necesario contextualizar el concepto de deuda ecológica en la relación comercio internacional-ambiente, revisando los postulados de diferentes autores sobre comercio justo y libre comercio.

El comercio internacional se relaciona con el ambiente en aspectos tales como:

- El efecto de las medidas de liberalización comercial, específicamente las distorsiones sobre los flujos del comercio internacional como reducciones de aranceles o eliminación de subsidios.

- Regulaciones ambientales que afectan las ventajas comparativas de las naciones y las decisiones de ubicación de plantas industriales.

Borrero considera:

La retórica aperturista y de libre comercio parece desconocer la situación actual de las relaciones internacionales, caracterizada por un gran proteccionismo en los países industrializados, por una relación de intercambio desfavorable para los países del Sur y por un aberrante flujo financiero del Sur hacia el Norte, contrariando todas las hipótesis de que el capital vendría del Norte si se creasen las condiciones apropiadas en el Sur (1994: 65).

Según Stiglitz (2005), el postulado que afirma desde Adam Smith (1776) y David Ricardo (1816) que el libre comercio sin trabas impuestas por el Estado mejora el 
bienestar social ha sido una doctrina altamente cuestionada, dado que los países no se enfrentan a la decisión de comercio libre, sino a la elección de diferentes grados de liberalización. El mismo autor señala:

Hoy prácticamente todos los países imponen algún tipo de restricción comercial o impuestos. Desde la II Guerra Mundial el mundo se ha ido moviendo gradualmente hacia la reducción de los aranceles y las restricciones al comercio. Algunos de los países desarrollados que se han mostrado como los más ardientes defensores de la liberalización comercial han sido en cierto modo hipócritas en su postura. Han negociado la reducción de aranceles y la eliminación de subvenciones para productos en los que ellos tienen una ventaja comparativa, pero son más reticentes a abrir sus propios mercados y a eliminar sus propias subvenciones en otras áreas en las que los países en desarrollo tienen la ventaja (Stiglitz, 2005: 39).

Por otra parte, existe un factor adicional que agudiza la desigualdad en el comercio internacional, el cual está relacionado, como lo expresa Herrera (2010), con el hecho de que los precios de los productos exportables de los países en desarrollo -productos primarios- históricamente han crecido menos que los precios de las manufacturas, maquinaria, equipos y tecnología importados.

Dadas las condiciones anteriormente planteadas, desde la economía ecológica se reconoce que el comercio internacional es un factor fuerte en la dinámica del creciente deterioro ambiental, pues, como lo indica Pérez (2006), se ha caído en una especie de "trampa" del subdesarrollo, donde a mayor crecimiento de los países industrializados mayores demandas de recursos naturales, lo que incentiva su explotación en los países en desarrollo. Esta situación, sumada a la obligación de pagar la deuda externa y sus intereses, lleva a una depredación mayor de la naturaleza. En términos de Martínez-Alier:

En efecto, para pagar la deuda externa y sus intereses hay que lograr un excedente (la producción ha de ser mayor que el consumo). Este excedente puede provenir por parte de un aumento genuino de la productividad (más 
producción por hora de trabajo), pero en parte sale del empobrecimiento de las personas de los países deudores y del abuso de la naturaleza. Mientras las deudas crecen, la naturaleza no puede crecer a un tipo de interés del cuatro o cinco por ciento anual, los recursos agotables, como el petróleo, no se producen sino que ya se produjeron hace tiempo, ahora se extraen y se queman, produciendo una variedad de efectos negativos; los recursos renovables tienen ritmos biológicos de crecimiento que son más lentos que esos ritmos económicos impuestos desde afuera (2001:31).

El libre comercio puede crear, a su vez, condiciones o ventajas competitivas, trasladando costos y carga ambiental a los países del Sur, mientras el Norte mantiene altos niveles de calidad ambiental dentro de sus fronteras (Muradian y Martinez-Alier, citados en Pérez, 2006: 7). En este contexto aparecen los conceptos de "paraísos de la contaminación" o de "paraísos contaminadores", para aquellos países aptos para industrias altamente contaminantes. Según Jenkins, "en la hipótesis de los paraísos de contaminadores se sugiere que, al liberalizar el comercio, en el hemisferio sur se tenderá a la especialización en industrias contaminantes, dada su legislación ambiental poco exigente" (2003: 87). Este mismo autor, citando a Jensen, explica:

En Jensen (1996) se analizan las distintas formulaciones de la hipótesis de los paraísos de contaminadores. Este autor señala que en varios estudios se hace hincapié en que usar deliberadamente una normativa ambiental débil para atraer capital equivale a implantar un paraíso de contaminación. Sin embargo, esta definición, que depende de las motivaciones de los responsables políticos, no puede comprobarse empíricamente mediante el análisis de la estructura del comercio internacional. Jensen ofrece una definición alternativa: dadas las diferencias políticas en cuanto a los niveles aceptables de emisiones, algunos países pueden obtener una ventaja comparativa al producir bienes con procesos industriales muy contaminantes (2003: 87).

Cuando la importancia de la dinámica de las relaciones de comercio internacional se asocia con la ciencia, debido a que el proceso productivo se va cualificando y aumentando, esta relación desencadena que no sólo exista una inquietud hacia 
el hacer, sino que introduce la importancia de analizar cada uno de los métodos implementados para la generación de herramientas útiles a unas necesidades planteadas por el ser humano.

Los procesos se intensificarán cada vez más, y el deficiente empoderamiento por indagar acerca de los métodos de producción ha conllevado durante el último siglo el indiscriminado uso de los recursos naturales. En este sentido, las actividades de mitigación y prevención no serán suficientes para equiparar la disponibilidad de recursos para un nuevo ciclo productivo.

Tal como lo expresan Ekins, Folke y Constanza, "la libertad para comerciar o no comerciar, dependiendo de si las condiciones son o no satisfactorias, se reemplaza con facilidad por una obligación de comerciar prácticamente sean cuales sean los términos del intercambio" (1994: 3).

Esta práctica puede tener gran explicación en la desagregación de los factores de producción: materia prima -que si provenía de un recurso natural se consideraba ilimitada - y mano de obra. Con el fin de la esclavitud, en términos de costos la mano de obra toma una relevancia excepcional: oficialmente se inaugura la carrera por construir, por crear y por demostrar cambios que faciliten la vida de las personas, lo que conlleva al auge y apogeo comercial. La consideración de los factores de producción conduce al análisis sobre el precio de intercambio. Así, cuando el precio es desigual en términos de costos ecológicos se puede hacer referencia al denominado dumping ecológico, "situación en la cual se fija el precio sin tener en cuenta el total de costos ecológicos y se vende por debajo de los costos totales de producción" (Alier et ál., 2001: 317).

Dentro de este contexto, el concepto de desarrollo sostenible refiere el "satisfacer la necesidades de las generaciones presentes sin comprometer la capacidad de las generaciones futuras para satisfacer sus propias necesidades" (Alier et ál.,2001:499). Este concepto tomó relevancia a través del informe Brundtland de 1987 y generó mayor conciencia sobre las implicaciones acerca de la escasez de los recursos y de cómo estos repercuten en el desarrollo de una sociedad mundial. El cambio en el imaginario respecto de lo que representa "necesidad", de acuerdo con los hábitos de consumo, se vuelve global, y los redireccionamientos en cuanto a los mecanismos 
de producción deben apuntar al aporte de soluciones estructurales, para que este desarrollo sea posible y sus relaciones de intercambio justas.

\section{Efecto escala del comercio internacional: multiplicador de la deuda ecológica}

\section{Existencia de la deuda ecológica}

Varios autores han desarrollado el concepto de deuda ecológica, el cual se origina en las intervenciones humanas y en los efectos del creciente comercio internacional y está caracterizado como desigual. Borrero afirma:

La deuda ecológica es una obligación con la biósfera por causa de intervenciones incompatibles en sus sistemas y procesos ecológicos esenciales, que han provocado destrucción de hábitats, extinción de especies y alteración grave de la homeostasis planetaria, con la consecuente amenaza para todas las formas vivientes.

La deuda ecológica es, también, una obligación con la humanidad, adquirida en razón de los daños, en ocasiones irreversibles, a la base biofísica de las sociedades, provocados por las islas de privilegio, economías del despilfarro e industrias de la barbarie, cuya consecuencia ha sido el empobrecimiento y exclusión de amplias mayorías humanas, etnocidio y avasallamiento de culturas (1994: 13).

La obligación con la biósfera es evidente, dado que la capacidad del planeta para absorber residuos y desechos que generan las actividades humanas es limitada y ha sido rebasada, tal como lo indican Azqueta, De la Cámara y Soltelsek: "El habitante promedio del planeta tiene una huella ecológica (unos patrones de consumo) que supera la capacidad de carga de la biósfera” (2005: 17). Por su parte, Robleto y Marcelo definen la deuda ecológica "como el patrimonio vital de la naturaleza que ha sido consumido y no restituido a ella" (citados en Borrero, 1994: 19). 
Este patrimonio incluye, según los autores, recursos naturales (especies y masas críticas) y condiciones ecológicas (pureza del aire, del agua y del atmosfera).

Según Martínez-Alier (2001) son varios los elementos de la deuda ecológica: un primer elemento de carácter económico, que parte del concepto de distribución ecológica espacial y está relacionado con el costo del uso desigual de los sumideros de carbono, no cancelado por aquellas economías cuyo alto grado de consumo emite una mayor cantidad de dióxido de carbono en comparación con el resto del mundo. Azqueta et ál. expresan: "En la esfera de las relaciones internacionales, esta distribución espacial del daño ambiental da lugar a un intercambio (ecológico) desigual mediante el que los países menos desarrollados tratan de mantener su competitividad" (2005: 17).

Muradian y Martínez-Alier consideran que

este comportamiento, por el que estos países degradan su medio ambiente para poder vender sus mercancías en los mercados internacionales, da lugar a una deuda ecológica en la que estarían incurriendo los países más desarrollados debido a la importación de estos productos, a unos precios "ecológicamente incorrectos" (citados en Azqueta et ál., 2005: 17).

En un sentido similar Goodland y Fonseca señalan, en primer lugar:

La contribución del Norte a los daños ambientales en el Sur es clara: el daño en la capa de ozono, el cambio climático, el efecto invernadero, la deforestación en los trópicos [...] El peligro creciente del fenómeno del calentamiento terrestre tendría una mayor incidencia en los trópicos, debido a la mayor sensibilidad a las variaciones y condiciones climáticas, y a las diferentes formas de contaminación que la sociedad está generando. Esta realidad configura una "deuda ecológica" creciente de los países industrializados, de alto consumo energético fósil, hacia los países subdesarrollados, poseedores de ecosistemas que se afectarían por este fenómeno (citados en Borrero, 1994: 20). 
Martinez-Alier (2001), en segundo lugar, señala la biopiratería, que hace referencia a la usurpación de prácticas indígenas sobre la cura o tratamiento de enfermedades, o a la apropiación ilegal de conocimientos locales sobre diversidad genética y biológica de la naturaleza.

Como tercer componente, el mismo autor habla de los pasivos ambientales, originados en la exploración y explotación de recursos fósiles. Como lo indican las conclusiones del Foro sobre deuda ecológica, organizado en Barrancabermeja, Santander, el 14 de julio de 2000, algunos de estos pasivos son: derrames y vertimientos de desechos que contaminan las fuentes hídricas, pérdida de agua para uso doméstico y agrícola, altas tasas de deforestación en las zonas de influencia petrolera, pérdida de biodiversidad, agotamiento irreversible de los recursos naturales, contaminación del recurso suelo - con la consiguiente disminución o pérdida total de productividad de grandes extensiones de tierra-, emisiones atmosféricas incontroladas, detrimento de la calidad de vida de los pobladores, pérdida de patrones culturales y tecnológicos de las comunidades de la zona (grupos étnicos o campesinos), generación de conflicto, violencia y ruptura del tejido social, problemas de salud en la comunidad, proliferación del consumismo, cambio de la vocación productiva de la región, entre otros.

\section{Efecto escala del comercio internacional}

Diversos autores han identificado un efecto escala del comercio internacional sobre el ambiente. Pérez considera:

La dinámica económica generada por el proceso de especialización productiva asociada al libre comercio internacional produce un efecto escala que incrementa la cantidad de los recursos naturales utilizados por la actividad económica y con ello la rematerialización de la economía mundial. Buena parte de estos recursos naturales son generados por las economías del sur, las cuales incrementan la exportación y explotación de sus recursos ambientales para soportar las relaciones de intercambio desiguales y el pago de sus compromisos financieros internacionales, incrementando con ello el efecto escala sobre su espacio ambiental (2006: 222). 
Dos factores originan el efecto escala, incrementando la deuda ecológica de los países deudores a los países acreedores: los aranceles y las subvenciones para productos en los que ellos tienen una ventaja comparativa y la presión de pago de la deuda externa.

Azqueta et ál. concluyen:

\begin{abstract}
Ahora bien, el problema del pago de una deuda acumulada por la imprevisión tanto de prestatarios como de prestamistas viene agravado por la dificultad de acceso a los mercados de los países desarrollados para multitud de productos primarios y manufacturados, en los que estos países tendrían una ventaja comparativa, debido a la presencia de una serie de barreras proteccionistas tanto arancelarias como no arancelarias (2005:3).
\end{abstract}

Según Coyler, esta condición inequitativa dificulta la obtención de divisas a través de la vía convencional de las ventajas comparativas, lo que conlleva a la búsqueda de nuevos yacimientos de recursos naturales (minerales, forestales, pesqueros) 0 a la sobreexplotación de los ya existentes (citado en Azqueta, 2005). Desde estas circunstancias la degradación ambiental es evidente.

Existe una circunstancia adicional en el llamado "comercio internacional desigual": la especialización productiva impuesta internacionalmente. Además de que los países menos favorecidos económicamente no pueden competir libremente con los productos que tradicionalmente pueden cultivar, los países industrializados demandan e imponen los productos que necesitan para garantizar su insostenible crecimiento y destructiva dinámica de consumo. Esta condición de especialización impuesta promueve el desarrollo de monocultivos, con todas las consecuencias que ampliamente los ambientalistas en todo el mundo han denunciado: pérdida de biodiversidad y conocimiento autóctono, fomento a las tecnologías y prácticas de la Revolución Verde y, en definitiva, un grave problema alimentario originado en la dependencia, dado que las transnacionales, las políticas y las fuentes de financiamiento controlan los componentes del sistema alimentario. 
Galeano ilustra con un ejemplo el problema alimentario:

Una nación cuya principal fuente alimentaria es el arroz está en dependencia cuando sus productores se ven obligados a adquirir la semilla, primer elemento del proceso productivo, a una empresa que ya monopoliza su consecución. Adicionalmente, depende de unas técnicas asociadas y específicas para la producción de esa semilla: uso de insumos externos vendidos por la misma empresa. Ahora bien, ¿qué pasará con esta nación cuando por un determinado conflicto o situación, la empresa que vende la semilla, los insumos y las técnicas no desee proporcionárselos más? El problema alimentario se liga enormemente a la soberanía alimentaria (2001: 54).

De igual forma, la pregunta entre la exportación y la importación debe ir más allá del precio de intercambio, ya que al importar, la preocupación va guiada al efecto sobre la dinámica comercial del país al cual ingresa el producto, pero no hay salvaguardias acerca de cómo se obtienen estos productos. Más en el interior del ciclo y a partir la teoría de Stakeholders (Freeman, 1984), para referirse a "quienes pueden afectar o son afectados por las actividades de una empresa": cualquier persona 0 entidad debe analizar cómo está afectando al país productor el extraer los recursos naturales para dicho ciclo productivo.

De acuerdo con lo anterior, los inconvenientes presentados pueden magnificarse a partir de los siguientes interrogantes, que plantearían el efecto escala del comercio internacional como multiplicador de la deuda ecológica: ¿qué bienestar puede ofrecer la nación a sus ciudadanos si la producción de arroz se realiza con el cultivo de organismos genéticamente modificados? ¿Qué pasará con la población de esta nación si las condiciones del mercado internacional demandan el arroz como insumo para la producción de biocombustibles?

Para llevar la reflexión al contexto nacional, se puede mencionar el caso del cultivo de caña. Existen menos exigencias en la etapa de establecimiento del cultivo con destino a la generación de biocombustibles para la producción de panela, lo cual va modificando la dinámica de oferta de este sector. 
En este sentido, AGROCADENAS afirma:

La panela ha tenido que enfrentar el deterioro de sus precios causado por la sobreoferta de producto, producida por la competencia del azúcar derretido y de las expectativas creadas por el uso de la caña en la producción de alcohol carburante, lo cual se pretende hacer a largo plazo en las zonas paneleras (2005: 2).

De igual forma, se ha visto el creciente cultivo de palma para biocombustible, desplazando cultivos de pancoger como yuca, ñame, plátano y hortalizas. Estos dos casos, que representan una oportunidad extensa de estudio, reflejan cómo se cierra la frontera entre la dinámica comercial y la seguridad alimentaria, aumentando la deuda ecológica. Por un lado, la obtención de biocombustible desplaza la producción de alimentos y, por otro, desencadena efectos sobre el suelo, que a largo plazo generan un pasivo incalculable, debido a que poseen la característica de ser extensivos y con un alto impacto de degradación por los químicos aplicados. Adicionalmente involucra una deficiencia en el valor monetario de intercambio, ya que los costos ambientales no son incluidos en la estructura de precio de la almendra ni del aceite de palma, pese a los esfuerzos por incluir indicadores de responsabilidad social.

De acuerdo con FEDEPALMA (2004), la producción de palma de aceite incrementó su contribución a la economía colombiana, pasando de participar en 1996 con el $5.6 \%$ del total de los cultivos permanentes, al 7.0\% en el 2000, cifra que evidencia el aumento por región para la producción y extensión de área sembrada en crecimiento.

\section{Reconocimiento de la creciente deuda ecológica: oportunidades para los países acreedores}

El reconocimiento de la existencia de la deuda ecológica en todos los actores, acreedores y deudores, es el primer desafío que enfrentan los denominados "países del Sur" para la formulación de estrategias que mitiguen los impactos del comercio internacional desigual en la biósfera. 
En este sentido, Vélez expresa:

Las demandas surgen de reconocer que los habitantes del Sur, sus territorios, sus relaciones sociales, su corporalidad y su oferta ambiental se ven erosionadas por una explotación irracional en nombre del desarrollo. El reclamo de la deuda ecológica es una expresión política de las reivindicaciones de los pueblos que han sido saqueados y conducidos al empobrecimiento material y cultural (2001: 42)

Sin embargo, Agarwal y Narain describen tres elementos que componen la posición que los países industrializados podrían asumir al respecto. En primer lugar, no reconocer las posibles obligaciones de perjuicios ocasionados en el pasado. En segunda instancia, elegir los problemas sobre los cuales el mundo entero debe trabajar, transfiriendo parte de la carga de los problemas ecológicos que mayoritariamente ellos ocasionaron. Y finalmente, no pagar como resultado de obligaciones adquiridas, sino brindar apoyo a los países acreedores para que no incurran en malas conductas ambientales (citados en Borrero, 1994).

El liderazgo del proceso de reconocimiento de la deuda ecológica es único y exclusivo de la sociedad civil. En palabras de Borrero:

El forjamiento de la solidaridad planetaria por la humanidad y por la biósfera no supondría la formalización a través de aparatos internacionales de una burocracia no gubernamental. Por el contrario, cada grupo o cada ciudadano en su propio espacio, en su localidad, en su trabajo, agenciará este espíritu de solidaridad planetaria (1994: 90).

Del reconocimiento se debe pasar al establecimiento de un instrumento para la defensa de los derechos e integridad del ambiente, mediante aproximaciones cualitativas y cuantitativas a la problemática. El diseño de esta herramienta va en dos sentidos: el primero, relacionado con la conservación y protección de la biósfera y el segundo, con la posibilidad de solicitar restauraciones y compensaciones por los daños causados. 
En lo relacionado con la conservación y protección de la biósfera, el camino propuesto es el de la recuperación de la soberanía alimentaria. Con el reconocimiento de la creciente deuda ecológica se evidencia la necesidad de retomar el derecho de la sociedad a alimentarse en vínculo con sus especificidades sociales, económicas, ambientales y culturales. Plantea Bravo:

[La soberanía alimentaria] va mas allá del concepto de seguridad alimentaria, que es el de asegurar una suficiente alimentación y segura para todos. Es velar por el autoabastecimiento alimentario a nivel local, regional y nacional. Pero también es la capacidad de tener control y decisión soberana en toda la red alimentaria, desde la producción de alimentos hasta el consumo (citada en Galeano, 2001: 69).

Vía Campesina (2001) aporta otro concepto de soberanía alimentaria, en concordancia con lo anteriormente señalado:

Es el derecho de cada pueblo a definir sus propias políticas agropecuarias y en materia de alimentación, a proteger y reglamentar la producción agropecuaria nacional y el mercado doméstico a fin de alcanzar metas de desarrollo sustentable, a decidir en qué medida quieren ser auto-suficientes, a impedir que sus mercados se vean inundados por productos excedentarios de otros países que los vuelcan al mercado internacional mediante la práctica del "dumping".

Finalmente, en cuanto a la opción de solicitar restauraciones y compensaciones por los daños causados por el comercio internacional a la biósfera, se presenta la oportunidad de canje de deuda externa por deuda ecológica. En este aspecto es importante aclarar que el canje no es deuda por naturaleza, dado que se ha evidenciado que en este intercambio median intereses económicos relacionados con la privatización de la biodiversidad biológica y con la apropiación de materias primas para los desarrollos biotecnológicos. En este caso se oculta una inversión con el seudónimo de "cancelación de deuda". Teniendo en cuenta que se están 
estimando valores de los daños causados, el intercambio es directo al costo de la deuda externa contabilizada.

\section{Conclusiones}

Toda actividad económica tiene origen en el uso intensivo de los recursos naturales. La deuda ecológica es consecuencia de la explotación irracional de la naturaleza, que se sintetiza en tres elementos: 1) el alto grado de emisión de contaminantes, debido al consumo de recursos fósiles para el sostenimiento del modelo económico impuesto por los países industrializados; 2) la biopiratería, relacionada con la apropiación abusiva, sistematización del conocimiento local y monopolización del saber popular; y 3) la generación de pasivos ambientales, originados en la explotación de recursos no renovables. La deuda ecológica es el daño causado a la biósfera y a la humanidad por la pérdida de servicios ambientales.

Considerando que el comercio internacional es desigual, un crecimiento de las transacciones induce al aumento del impacto ambiental, a través del incremento en la cantidad de recursos materiales movilizados. Este fenómeno se conoce como efecto escala del comercio internacional, que, a su vez, se constituye como multiplicador de la deuda ecológica. Ante esta situación, para alcanzar un modelo económico internacional más sostenible se debe considerar la cantidad de material, energía y territorio incorporado.

El reconocimiento de la deuda ecológica - proceso difícil de realizar, dada la posición dominante de los países deudores- es primordial en el establecimiento de una herramienta política con dos objetivos: 1) la conservación y protección de la biósfera, a través de la recuperación de la soberanía alimentaria; 2) la posibilidad de canjear la deuda externa por el pago de restauraciones y compensaciones en respuesta a los daños causados.

El canje de la deuda externa a través del reclamo de la deuda ecológica sobrepasa a las necesidades de modificación de las políticas económicas, ya que éste es un concepto que se adentra hacia cómo se percibe el mundo a través de las necesidades de consumo y de cómo los países deudores se hacen responsables de su impacto 
para el futuro. Es incalculable la deuda con la que por defecto llega un nuevo ser al mundo, producto de siglos caracterizados por la proliferación de actividades que requieren del consumo intensivo y no restituible de recursos naturales.

Teniendo en cuenta las anteriores conclusiones, se hace necesaria una reestructuración en la fijación de precios de intercambio y configuración del costo de producción a partir del uso de los recursos naturales. Se encuentra abierto el tema hacia la investigación, con el fin de realizar el trabajo de campo requerido para reconfigurar los elementos cualitativos que determinan la forma de producción e insertar dentro de la dinámica comercial los factores que hasta el momento se han venido asimilando como externalidades (impactos) y que reflejan el carácter determinante que poseen para el asertivo direccionamiento de la deuda ecológica.

\section{Referencias}

Azqueta, De la Cámara y Sotelsek (s.f.). Degradación ambiental, endeudamiento externo y comercio internacional. Universidad de Alcalá, Departamento de Fundamentos de Economía e Historia Económica.

Ball, Kothari y Robin (2000). The Effect of International Institutional Factors on Properties of Accounting Earnings. Journal of Accounting and Economics, 29(1), 1-51.

Borrero Navia,J.(1994). La deuda ecológica. Testimonio de una reflexión. Cali: Feriva.

Ekins, Folke y Constanza (1994). Trade, Environment and Development: The Issues in Perspective. Ecological Economist, 9.

Epstein, J. (2008). El desempeño ambiental en la empresa. Bogotá: Ecoe.

FEDEPALMA-CENIPALMA (2004). Anuario estadístico 2004. La agroindustria de la palma de aceite en Colombia y el mundo 1999-2003. Bogotá.

Freeman, E. (1984). Strategic Management: A Stakeholder Approach. Pitman Series in Business and Public Policy. 
Gómez, C.E.(2002).Liberalización comercial y su impacto sobre el medio ambiente y los recursos naturales: un enfoque de equilibrio general. (Tesis doctoral). Universidad de Alcalá, Departamento de Fundamentos de Economía e Historia Económica.

Gray, R. (2003). Contabilidad y auditoría ambiental. Bogotá: Ecoe.

Herrera Valencia, B. (2010). Fomentar una alianza mundial para el desarrollo. "Que el sistema económico y jurídico funcione". En Los objetivos de desarrollo del milenio. Un compromiso con los derechos económicos, sociales y culturales. Bogotá: Anthropos.

Jenkins, R. (2003). La apertura comercial ¿ha creado paraísos de contaminadores en América Latina? Revista de la CEPAL, 80, 85-100.

Observatorio Agrocadenas Colombia (2005). La cadena agroindustrial de la panela en Colombia: una mirada global de su estructura y dinámica 1991-2005. Bogotá: Ministerio de Agricultura y Desarrollo Rural.

Pérez Rincón, M.A. (2006). Comercio internacional y medio ambiente en Colombia. (Tesis doctoral). Barcelona: Universidad Autónoma de Barcelona, Institut de Ciènci Tecnologia Ambientals (ICTA).

Martínez-Alier, J. (2001). La deuda ecológica. En Una exigencia del Sur: reconocer la deuda ecológica. Bogotá: Editorial Bochica.

Martínez-Alier, J.y Jusmet Roca, J. (2006). Economía ecológica y política ambiental. México: Fondo de Cultura Económica.

Stiglitz, J. (2005). El comercio puede ser bueno para el desarrollo. En Comercio justo para todos. Bogotá: Aguilar.

Vía Campesina (2001). Soberanía alimentaria. Recuperado de http://www.viacampesina.org/sp/ 